\title{
A Nekhoroshev-Type Theorem for Hamiltonian Systems with Infinitely Many Degrees of Freedom
}

\author{
Giancarlo Benettin, ${ }^{1}$ Jürg Fröhlich ${ }^{2}$ and Antonio Giorgilli ${ }^{3}$ \\ 1 Dipartimento di Fisica dell'Università di Padova, Via Marzolo 8, I-35131 Padova, Italy \\ 2 Theoretical Physics, ETH-Hönggerberg, CH-8093 Zürich, Switzerland \\ 3 Dipartimento di Fisica dell'Università di Milano, Via Celoria 16, I-20133 Milano, Italy
}

\begin{abstract}
We study the propagation of lattice vibrations in models of disordered, classical anharmonic crystals. Using classical perturbation theory with an optimally chosen remainder term (i.e. a Nekhoroshev-type scheme), we are able to show that vibrations corresponding to localized initial conditions do essentially not propagate through the crystal up to times larger than any inverse power of the strength of the anharmonic couplings.
\end{abstract}

\section{Introduction: General Ideas and Main Results}

In this paper we study the trapping, or localization of lattice vibrations in models of classical, disordered anharmonic crystals. Localization is a phenomenon observed in the study of wave propagation in disordered media. When the disorder is large enough waves can get trapped or propagate anomalously slowly. This implies that there is no transport of energy in such a system.

Anderson first studied localization in the motion of quantum mechanical electrons through a random array of scatterers [1]. This is relevant for an analysis of electrical conductivity in disordered metals. Anderson's arguments have been made rigorous in [2] (for one-dimensional systems) and [3] (higher dimensional systems). Electron waves are linear waves whose propagation is governed by the Schrödinger equation. In contrast, the transport of heat or sound through bulk matter, or of electromagnetic waves through a non-linear medium, poses a problem in non-linear wave propagation. One would like to understand, for example, the transport properties of a disordered, anharmonic crystal. A conventional idea is that if the disorder is large enough there is no transport of energy in such a system, or transport is anomalously slow. A proof of this conjecture would require mathematical control of wave propagation over arbitrarily long time scales. For a special class of models of classical anharmonic crystals and some very special initial conditions, such control has been achieved in [4]. But the results in [4] do not permit one to establish bulk transport properties of those model systems.

Real or computer experiments are performed in a finite, though possibly long time interval. One might argue, therefore, that it is of interest to study wave 
propagation over long, but finite time intervals. Consider, for example, a classical, anharmonic disordered crystal with a Hamilton function that is a small perturbation, measured by a parameter $\varepsilon$, of a quadratic Hamilton function. The disorder in the system renders the frequencies of the harmonic vibrations non-resonant, with large probability, and those vibrations remain localized for all time. One may therefore expect that vibrations of the anharmonic crystal with localized initial conditions remain localized up to times $T(\varepsilon)$, with $T(\varepsilon) \rightarrow \infty$, as $\varepsilon \rightarrow 0$, faster than any inverse power of $\varepsilon$. Such a result would explain, to some extent, the absence of transport encountered in real or computer experiments performed on those systems.

It is the purpose of our paper to rigorously establish localization of vibrations in classical disordered crystals which are perturbations of strength $\varepsilon$ of disordered, harmonic crystals, up to times larger than any inverse power of $\varepsilon$. Our method of proof is classical perturbation theory which is applicable, because disorder renders resonances highly improbable. We shall construct functionals on phase space which are integrals of the motion up to some order $M$ in the strength $\varepsilon$ of the anharmonic perturbation. Our main result follows from an optimal choice of $M$, depending on $\varepsilon$. This method is inspired by the work of Nekhoroshev $[5,6]$.

We now introduce the models studied in this paper: we consider an infinite array of oscillators, one at each site of a $d$-dimensional lattice, here chosen to be $\mathbf{Z}^{d}$. These oscillators are coupled by an anharmonic interaction. In standard canonical coordinates $p=\left\{p_{s}\right\}_{s \in \mathbf{Z}^{d}}, q=\left\{q_{s}\right\}_{s \in \mathbf{Z}^{d}}$, the unperturbed Hamilton function, $\mathrm{H}_{2}$, is given by

$$
H_{2}(p, q)=\frac{1}{2} \sum_{s \in \mathbf{Z}^{d}} \omega_{s}\left(p_{s}^{2}+q_{s}^{2}\right)
$$

where $\omega=\left\{\omega_{s}\right\}_{s \in \mathbf{Z}^{d}}$ is a family of infinitely many independent, identically distributed random variables. For the sake of concreteness we choose the distribution of a single frequency, $\omega_{s}$, to be Gaussian, i.e.

$$
d \rho\left(\omega_{s}\right)=\frac{1}{\sigma \sqrt{\pi}} e^{-\omega_{s}^{2} / \sigma} d \omega_{s},
$$

but our methods extend to a rather general class of $d \rho$ 's.

The anharmonic perturbation, $H_{I}$, is a sum of polynomials $V_{X}$, where $X$ is an arbitrary finite subset of $\mathbf{Z}^{d}$, and $V_{X}$ only depends on $\left\{p_{s}, q_{s}\right\}_{s \in X}$. Moreover, $V_{X}$ is exponentially small in the cardinality, $|X|$, of $X$ and becomes small rapidly in the diameter of $X$. It need, however, not be of finite range. It has been shown in [4] that rather realistic models of classical, anharmonic disordered crystals can be cast in this form. (This follows from the methods of [3].)

For simplicity we shall only consider two-body interactions $(|X|=2)$ which are cubic in $q$ and independent of $p$. But we should emphasize that our techniques apply to much more general interactions.

Denoting by $\mathscr{L}$ the set of unordered pairs of sites in $\mathbf{Z}^{d}$, called lattice lines, the interaction Hamiltonian can be written in the form

$$
H_{I}(p, q)=H_{3}(p, q)=\sum_{l \in \mathscr{L}} V_{l}\left(q_{s^{\prime}}, q_{s^{\prime \prime}}\right)
$$


where $s^{\prime}, s^{\prime \prime}$ are the ends of the line $l$, and $V_{l}$ is a cubic polynomial in $q_{s^{\prime}}, q_{s^{\prime \prime}}$; as will be explained more precisely later, $V_{l}$ will be required to decay rapidly with the length of $l$. The complete Hamiltonian is then

$$
H(p, q)=H_{2}(p, q)+H_{3}(p, q) .
$$

Assume our system is given a small initial excitation localized around the (arbitrarily chosen) origin, i.e. we choose initial conditions satisfying

$$
\left(p_{s}^{2}+q_{s}^{2}\right)^{1 / 2} \leqq \varepsilon \psi(|s|), \quad|s|=\sum_{i=1}^{d}\left|s_{i}\right|,
$$

where $\psi$ is a suitable function, which decays to zero for large $|s|$; the precise form of $\psi$ will be introduced later. Our aim is to prove an estimate from below for the time-scale at which the initial excitation significantly evolves and, in particular, delocalizes.

We define $\Phi_{2}^{s}(p, q)=\frac{1}{2}\left(p_{s}^{2}+q_{s}^{2}\right)$, so that $H_{2}=\sum_{s \in Z^{d}} \omega_{s} \Phi_{2}^{s}$. Clearly, for each $s \in \mathbf{Z}^{d}$, $\Phi_{2}^{s}$ is an integral of motion of $H_{2}$, and also an approximate integral for the complete Hamiltonian: Indeed, the Poisson bracket $\left\{H_{3}, \Phi_{2}^{s}\right\}$ is cubic, so that

$$
\dot{\Phi}_{2}^{s}=\left\{H, \Phi_{2}^{s}\right\}=\left\{H_{3}, \Phi_{2}^{s}\right\}=\mathcal{O}\left(\varepsilon^{3}\right)
$$

and since $\Phi_{2}^{s}$ is only of order $\varepsilon^{2}$, it needs a rather long time-scale $\mathscr{C}\left(\varepsilon^{-1}\right)$ to significantly evolve. To go beyond this first-order result, one can work perturbatively, and look for almost-integrals in the form of polynomials of higher order, say

$$
\Phi^{s, M}(p, q)=\sum_{m=2}^{M} \Phi_{m}^{s}(p, q), \quad M \geqq 3,
$$

where $\Phi_{m}^{s}(p, q)$ is a homogeneous polynomial of order $m$ in $p$ and $q ; \Phi^{s, M}$ should be localized in some sense around the site $s \in \mathbf{Z}^{d}$, and satisfy

$$
\dot{\Phi^{s . M}} \equiv\left\{H, \Phi^{s, M}\right\}=\mathcal{O}\left(\varepsilon^{M+1}\right) .
$$

If one succeeds in this program, then one expects that any initial excitation localized around the origin takes a time of order $\varepsilon^{-M+1}$ to significantly evolve and delocalize. Moreover, following Nekhoroshev [5,6] (see also [7-10]), one can attempt, for small $\varepsilon$, to go beyond any finite order in $\varepsilon$; this means one wants the order $M$ to be a function of $\varepsilon$, with $M \rightarrow \infty$ for $\varepsilon \rightarrow 0$. In the finite dimensional case, it is possible to let $M$ grow like an inverse power of $\varepsilon$, and get time-scales of oder $\exp \left(\varepsilon_{0} / \varepsilon\right)^{a}, \varepsilon_{0}$ and $a$ being suitable positive constants (however, $\varepsilon_{0}$ and $a$ depend on the number of degrees of freedom, and vanish as $n \rightarrow \infty$ ). In our infinite-dimensional problem, there are some essential difficulties in proving such a result, and we are only able to control the motion up to time-scales of the form

$$
\exp \frac{a\left(\ln \varepsilon^{-1}\right)^{2}}{\ln \ln \varepsilon^{-1}}
$$

which still grow faster than any finite power of $\varepsilon^{-1}$, but are definitely much shorter than Nekhoroshev-like time scales.

In order to provide a formal statement, let us define precisely what we 
mean by "rapid decay" of $V_{l}$. For any lattice line $l$ with ends in $\mathbb{Z}^{d}$, with projections $l_{1}, \ldots, l_{d}$ onto the lattice axes, let $|l|=\left|l_{1}\right|+\cdots+\left|l_{d}\right|$. Denoting $V_{l}\left(q_{s^{\prime}}, q_{s^{\prime \prime}}\right)=$ $\sum_{j=0}^{3} v_{l, j} q_{s^{\prime}}^{j} q_{s^{\prime \prime}}^{3-1}$, we say that $V_{l}$ decays rapidly, if there exist positive constants $U$, $K, \delta$, with $\delta \leqslant 1$, such that one has

$$
\sum_{j=0}^{3}\left|v_{l, j}\right| \leqq U e^{-K(1+\mid l !)^{\delta}}
$$

We also introduce the notations $\psi(x)=(1+x)^{-1-d}$, and $\kappa=\sum_{s \in Z^{d}} \psi(|s|)$.

Our main result is the following theorem.

Theorem. Let

$$
H=\sum_{s \in \mathbf{Z}^{d}} \omega_{s} \Phi_{2}^{s}+\sum_{l \in \mathscr{L}} V_{l},
$$

as defined above. Assume that $\left\{\omega_{s}\right\}_{s \in \mathbf{Z}^{d}}$ are independent, identically distributed gaussian random variables, with variance $\sigma$, and that $V_{l}$ decays rapidly with $|l|$, as defined in (1.10). Then there exist a set of frequencies $\Omega$, with mes $\Omega<\gamma(\kappa / \sigma \sqrt{\pi})$, and two constants $\mathscr{T}, \mathscr{G}$, depending on $d, U, K, \delta, \gamma$, such that, for $\omega \notin \Omega$, and any initial condition $\left(p^{0}, q^{0}\right)$ satisfying

$$
\Phi_{2}^{s}\left(p^{0}, q^{0}\right) \leqq \frac{1}{2} \varepsilon^{2} \psi(|s|)^{2}, \quad s \in \mathbf{Z}^{d},
$$

with \& small, one has

$$
\left|\Phi_{2}^{s}(p(t), q(t))-\Phi_{2}^{s}\left(p^{0}, q^{0}\right)\right| \leqq \mathscr{G}_{\varepsilon}^{3} \psi(|s|)^{3}, \quad s \in \mathbf{Z}^{d},
$$

for

$$
|t| \leqq \mathscr{T} e^{(1 / 8)\left(\ln \varepsilon^{-1}\right)^{2} / \ln \ln \varepsilon^{-1}}
$$

The proof of the theorem is contained in the next three sections. In Sect. 2, we explain the algebraic scheme of our perturbative construction. In Sect. 3 we show how one can introduce rigorous estimates in our infinite-dimensional problem. Finally, in Sect. 4, we obtain the main estimate and conclude the proof of the theorem.

\section{The Algebraic Construction}

Denote by $\Pi_{m}$ the set of homogeneous polynomials of degree $m$ in $p_{s}, q_{s}, s \in \mathbf{Z}^{d}$, depending only on a finite subset of coordinates. Our purpose is to construct, for each $s \in \mathbf{Z}^{d}$, a polynomial $\Phi^{s . M}(p, q)=\sum_{m=2}^{M} \Phi_{m}^{s}(p, q)$, with $M \geqq 3$ and $\Phi_{m}^{s} \in \Pi_{m}$, for $m=2, \ldots, M$, such that

$$
\left\{H, \Phi^{s, M}\right\} \in \Pi_{M+1} \text {. }
$$

Since $H=H_{2}+H_{3}$ and $\left\{H_{2}, \Phi_{2}^{s}\right\}=0$, this equation is immediately seen to be equivalent to the $M-2$ equations

$$
\left\{H_{2}, \Phi_{m}^{s}\right\}+\mathscr{R}_{m}^{s}=0, \quad m=3, \ldots, M,
$$


with

$$
\mathscr{R}_{m}^{s}=\left\{H_{3}, \Phi_{m-1}^{s}\right\}
$$

indeed, if these equations can be solved, then one has

$$
\left\{H, \Phi^{s, M}\right\}=\left\{H_{3}, \Phi_{M}^{s}\right\} \in \Pi_{M+1} .
$$

Each of the equations (2.2) should be regarded as an equation for $\Phi_{m}^{s}, \mathscr{R}_{m}^{s} \in \Pi_{m}$ being a known term. Clearly, one can solve these equations recursively, as far as one is able to invert the linear operator $L=\left\{H_{2}, \cdot\right\}$ inside $\Pi_{m}$; for this purpose, one must show that $\mathscr{R}_{m}^{s}$ has no components in the null space, $\Pi_{m}^{0}$, of $L$.

Now, as is well known, the operator $L$, acting on polynomials with finitely many variables, is diagonalizable: if one introduces complex canonical variables

$$
x_{s}=\frac{1}{\sqrt{2}}\left(p_{s}+i q_{s}\right), \quad y_{s}=-\frac{i}{\sqrt{2}}\left(p_{s}-i q_{s}\right),
$$

then $L$ takes the form

$$
L=i \sum_{s \in Z^{d}} \omega_{s}\left(x_{s} \frac{\partial}{\partial x_{s}}-y_{s} \frac{\partial}{\partial y_{s}}\right) .
$$

It is then clear that a basis of eigenfunctions in $\Pi_{m}$ is given by $u_{j k}(x, y)=x^{j} y^{k}$, for any $j, k \in \mathbf{N}^{\mathbf{Z}^{d}}$ with $\sum_{s \in \mathbf{Z}^{d}}\left(j_{s}+k_{s}\right)=m ;(\mathbf{N}$ denotes here the set of non-negative integers). The corresponding eigenvalue is $i \omega \cdot(j-k)$, so that the null space $\Pi_{m}^{0}$ is spanned by those functions $u_{j k}$, for which $\omega \cdot(j-k)=0$.

We now restrict our attention to the so-called non-resonant case, i.e., we consider frequencies $\omega=\left\{\omega_{s}\right\}_{s \in \mathbf{Z}^{d}}$, such that

$$
\omega \cdot v=0 \Rightarrow v=0,
$$

for each $v \in \mathbf{Z}^{\mathrm{z}^{d}}$ of compact support; in this case $\Pi_{m}^{0}$ is spanned by functions of the form $u_{j j}=(i / 2) \Pi_{s}\left(p_{s}^{2}+q_{s}^{2}\right)^{j_{s}}, j \in \mathbf{Z}^{\mathbf{Z}^{d}}$.

A very elementary argument, taken from [11], shows that in this non-resonant case, the terms $\mathscr{R}_{m}^{s}, m=3, \ldots, M$, cannot have components in $\Pi_{m}^{0}$, so that Eq. (2.2) for $\Phi_{m}^{s}$ can be solved. Indeed, first of all it is clear that $\Pi_{m}^{0}, m=3, \ldots, M$, contains only functions which are even in $p$. Since $H_{3}$ and $\Phi_{2}^{s}$ are even in $p$, $\mathscr{R}_{3}^{s}=\left\{H_{3}, \Phi_{2}^{s}\right\}$ is odd, and cannot have components in $\Pi_{3}^{0}$ : consequently, the first equation can be solved for $\Phi_{3}^{s}$, and since $\left\{H_{2}, \Phi_{3}^{s}\right\}$ is odd, with $H_{2}$ even, $\Phi_{3}^{s}$ is necessarily even. Proceeding by induction, one finds that, for any $m=3 \ldots, M, \mathscr{R}_{m}^{s}$ is odd in $p$, and all equations can be solved, with the solutions, $\Phi_{m}^{s}$, even in $p$.

Using the complex coordinates $x$ and $y$, and introducing the notation

$$
\mathscr{R}_{m}^{s}=\sum_{j, k \in \mathbf{N}^{d}} \mathscr{R}_{m \cdot j k}^{s} x^{j} y^{k}
$$

the solution of (2.2) can be written in the form

$$
\Phi_{m}^{s}=\sum_{j, k \in \mathbb{Z}^{d}} \frac{\mathscr{R}_{m, j k}^{s}}{i \omega \cdot(j-k)} x^{j} y^{k} .
$$




\section{Small Denominators and Diagrams}

Our aim is now to supplement the formal scheme outlined in the previous section by rigorous estimates. For this purpose, for a generic polynomial $f \in \Pi_{m}$ written in the form

$$
f=\sum_{j, k \in \mathbf{N} Z^{d}} f_{j k} x^{j} y^{k},
$$

we will make use of the norm

$$
\|f\|=\sum_{l, k \in \mathbf{N}^{d}}\left|f_{j k}\right|
$$

In the finite-dimensional case, it is not too difficult [12] to produce efficient recursive estimates for the quantities $\mathscr{R}_{m}^{s}$ and $\Phi_{m}^{s}$ entering Eq. (2.2). The scheme is the following: First of all, one makes use of the definition (2.3) of $\mathscr{R}_{m}^{s}$ to estimate $\left\|\mathscr{R}_{m}^{s}\right\|$, assuming $\left\|H_{3}\right\|$ and $\left\|\Phi_{m-1}^{s}\right\|$ are already estimated; then, using (2.9), one can write

$$
\left\|\Phi_{m}^{s}\right\|=\alpha_{m}^{-1}\left\|\mathscr{R}_{m}^{s}\right\|
$$

where $\alpha_{m}$ is the absolute value of the smallest denominator appearing in (2.9). Finally, to estimate $\alpha_{m}$, one usually introduces a Diophantine condition on $\omega$ of the form

$$
|\omega \cdot v| \geqq \gamma|v|^{-n}
$$

$n$ being the number of degrees of freedom. In fact, on the one hand such a condition is satisfied by a large set of frequencies, while, on the other hand, when used in (3.3), it still prevents too fast an explosion of the estimates.

This simple scheme poses some essential difficulties in our problem with infinitely many degrees of freedom: it is not easy to replace (3.4) by another condition, which, although satisfied by a set of frequencies of large Gaussian measure, leads nevertheless to reasonable estimates. The way out we propose is based on a rather finer analysis of the solution (2.9) of Eq. (2.2); the main idea is to replace estimates of the form (3.3), which are rather crude because they treat all small denominators uniformly, by finer ones, where different terms, with different small denominators, are estimated separately. To properly classify the terms, we introduce a convenient diagrammatic technique.

Let $\mathscr{L}$ denote the set of lattice lines, i.e. the (non-oriented) segments with ends on lattice sites. If $l \in \mathscr{L}$ has projections of length $l_{1}, \ldots, l_{d}$ on the lattice axes, then we let $|l|=l_{1}+\cdots+l_{d}$. Lines $l$ of null length are also included. Denote by $D$ any finite connected diagram, i.e. a finite connected set of lines belonging to $\mathscr{L}$ (the same line can appear several times). Denote by $|D|$ the number of lines in $D$, and by $\mathscr{S}(D)$ the support of $D$ in $\mathbf{Z}^{d}$, i.e., the set of ends of the lines of $D$. Finally, denote by $\mathscr{D}^{s}$ the set of diagrams such that $s \in \mathscr{S}(D)$, and by $\mathscr{D}_{m}^{s}$ the subset $\left\{D \in \mathscr{D}^{s} ;|D|=m\right\}$. For later notational convenience, we also include in $\mathscr{D}^{s}$ a diagram $D$ with zero lines, assuming, for this diagram, $\mathscr{S}(D)=\{s\}$; the set $\mathscr{D}_{0}^{s} \subset \mathscr{D}^{s}$ is intended to contain just this diagram.

Our purpose is to write the polynomials $\Phi_{m}^{s} \in \Pi_{m}$ we are dealing with, in the form

$$
\Phi_{m}^{s}=\sum_{D \in \mathscr{C}_{m-2}^{s}} \Phi_{m, D}^{s}
$$


where $\Phi_{m, D}^{s}$ is a homogeneous polynomial of degree $m$ in $p_{t}$ and $q_{t}, t \in \mathscr{S}(D)$. For $m=2, \Phi_{2}^{s}$ already has this form; thus, assuming that $\Phi_{m}^{s}$ has this form for $m \geqq 2$, let us show that $\Phi_{m+1}^{s}$ can also be written in this form.

As already remarked, the interaction Hamiltonian has the form $H_{3}=\sum_{l \in \mathscr{L}} V_{l}$, where $V_{l}$ depends only on the variables attached to the ends of $l$. From (2.3) one then has

$$
\mathscr{R}_{m+1}^{s}=\sum_{D^{\prime} \in \mathscr{S}_{m-2}^{s}} \sum_{l \in \mathscr{L}}\left\{V_{l}, \Phi_{m, D^{\prime}}^{s}\right\}
$$

and, clearly, the Poisson bracket is different from zero only if at least one of the ends of $l$ belongs to $\mathscr{S}\left(D^{\prime}\right)$, i.e., if $D^{\prime} \cup\{l\}$ is connected, and thus belongs to $\mathscr{D}_{m-1}^{s}$. One then has

with

$$
\mathscr{R}_{m+1}^{s}=\sum_{D \in \mathscr{\mathscr { T }}_{m-1}^{s}} \mathscr{R}_{m+1, D}^{s}
$$

$$
\mathscr{R}_{m+1, D}^{s}=\sum_{\substack{l \in D \\ D \backslash\{l\} \in \mathscr{T}_{m-2}^{s}}} \frac{1}{\mu(l, d)}\left\{V_{l}, \Phi_{m, D \backslash\{l\}}^{s}\right\},
$$

where $\mu(l, D)$ is the multiplicity of the line $l$ in the diagram $D$ (which must be introduced to avoid overcounting). It immediately follows that $\Phi_{m+1}^{s}=-L^{-1} \mathscr{R}_{m+1}^{s}$ can be written in the form (3.5), with $m+1$ in place of $m$, and

$$
\Phi_{m+1, D}^{s}=-L^{-1} \mathscr{R}_{m+1, D}^{s}
$$

(Let us notice that $L^{-1}$ does not alter the order of the polynomials, nor does it introduce new variables, so that $\Phi_{m+1, D}^{s}$ has indeed all the required properties.)

The idea is now to work out rigorous estimates for $\mathscr{R}_{m}^{s}, m=3, \ldots, M+1$, proceeding recursively as in the finite-dimensional case, but keeping track, at each step, of the diagrammatic classification appearing in (3.7), (3.9). To begin with, let us first prove the following elementary lemma.

Lemma 1. If $f \in \Pi_{m}$ and $f^{\prime} \in \Pi_{m^{\prime}}$, then one has $\left\|\left\{f, f^{\prime}\right\}\right\| \leqq m m^{\prime}\|f\|\left\|f^{\prime}\right\|$.

Proof. If $f=\sum_{j k} f_{j k} x^{j} y^{k}, f^{\prime}=\sum_{j^{\prime} k^{\prime}} f_{j^{\prime} k^{\prime}}^{\prime} x^{j^{\prime}} y^{k^{\prime}}$, then one has

$$
\left\{f, f^{\prime}\right\}=\sum_{j k j^{\prime} k^{\prime}} f_{j k} f_{j^{\prime} k^{\prime}}^{\prime} \sum_{s \in Z^{d}}\left(j_{s} k_{s}^{\prime}-j_{s}^{\prime} k_{s}\right) x^{j-e_{s}} y^{k-e_{s}},
$$

where $e_{s} \in \mathbf{N}^{\mathbf{Z}^{d}}$ has all components vanishing except the one corresponding to the site $s$, which has the value 1 . One then has

$$
\left\|\left\{f, f^{\prime}\right\}\right\| \leqq \sum_{j k j^{\prime} k^{\prime}}\left|f_{j k}\right|\left|f_{j^{\prime} k^{\prime}}^{\prime}\right| \sum_{s \in Z^{d}}\left(j_{s} k_{s}^{\prime}+j_{s}^{\prime} k_{s}\right) .
$$

Now, since $j_{s}, k_{s} \leqq m$, one can write $\sum_{s}\left(j_{s} k_{s}^{\prime}+k_{s} j_{s}^{\prime}\right) \leqq m \sum_{s}\left(j_{s}^{\prime}+k_{s}^{\prime}\right)=m m^{\prime}$, and the conclusion is immediate.

This lemma can be applied to Eq. (3.8), to get the estimate

$$
\left\|\mathscr{R}_{m+1, D}^{s}\right\| \leqq \sum_{\substack{l \in D \\ D \backslash\left\{\{\} \in \mathscr{T}_{m-2}^{s}\right.}} 3 m\left\|V_{l}\right\|\left\|\Phi_{m, D \backslash\{l\}}^{s}\right\|
$$


(the multiplicity of lines in the diagram $D$ has been disregarded, as it hardly could be used to improve the estimates).

We now pass to the most crucial step: We introduce our Diophantine condition for $\omega$. The idea is that, for each diagram, more precisely for all $s \in \mathbf{Z}^{d}, m \geqq 3$, and for any $D \in \mathscr{D}_{m-2}^{s}$, one writes the condition in the form

$$
|\omega \cdot v|>\alpha_{m, D}^{s} \quad \forall|v| \leqq m, \operatorname{supp} v \subseteq \mathscr{S}(D) ;
$$

the numbers $\alpha_{m, D}^{s}$ are chosen as large as possible (in order to have good estimates) with however the requirement that condition (3.13) is satisfied by a set of $\omega$ 's of large measure. Our choice is the following:

$$
\alpha_{m, D}^{s}=\frac{\gamma}{(1+|s|)^{d+1}} \frac{(8 \kappa)^{-(m-2)}}{(m-2) !} \prod_{l \in D} \frac{1}{(1+|l|)^{d+1}},
$$

where $\gamma$ is a (small) positive constant, while $\kappa$ denotes the sum of the convergent series $\sum_{s \in \mathbf{Z}^{d}}(1+|s|)^{-d-1}$. With this choice, the set of frequencies which do not satisfy condition (3.13) has measure of order $\gamma$, as guaranteed by the following lemma.

Lemma 2. The set, $\Omega$, of frequencies $\omega \in \mathbf{R}^{Z^{d}}$ which do not satisfy condition (3.13), with $\alpha_{m, D}^{s}$ given by (3.14), has gaussian measure estimated by

$$
\operatorname{mes} \Omega<\frac{\gamma \mathcal{K}}{\sigma \sqrt{\pi}}
$$

where $\sigma$ is the variance of the gaussian distribution.

Proof. For $s \in \mathbf{Z}^{d}, m \geqq 3, D \in \mathscr{D}_{m-2}^{s}, v \in \mathbf{Z}^{\mathrm{Z}^{d}}$ with $\operatorname{supp} v \subseteq \mathscr{S}(D)$, denote

$$
\Omega_{m, D}^{s}(v)=\left\{\omega \in \mathbf{R}^{\mathbf{Z}^{d}} ;|\omega \cdot v|<\alpha_{m, D}^{s}\right\} ;
$$

then one has

$$
\operatorname{mes} \Omega \leqq \sum_{s} \sum_{m} \sum_{D} \sum_{v} \operatorname{mes} \Omega_{m, D}^{s}(v) .
$$

The measure of $\Omega_{m, D}^{s}(v)$ is easily estimated by

$$
\operatorname{mes} \Omega_{m, D}^{s}(v) \leqq \frac{1}{\sigma \sqrt{\pi}} \| \frac{\alpha_{m, D}^{s}}{\|v\|_{e}} \leqq \frac{\alpha_{m, D}^{s}}{\sigma \sqrt{\pi}},
$$

where $\|v\|_{e}$ is the euclidean length of $v$. This follows from the invariance of the Gaussian distribution under rotations, by integrating out all components but the one corresponding to the direction orthogonal to $v$.

To perform the sum over $v$, one must recall that the number, $J_{n, m}$, of integer vectors $v \in \mathbf{Z}^{n}$, with $|v|=m$, is $\left(\begin{array}{c}m+n-1 \\ m\end{array}\right)$, as is easily seen by recurrence; in our case $n$ is the cardinality of $\mathscr{S}(D)$, which is $|D|+1=m-1$, and thus $J_{n, m}=\left(\begin{array}{c}2 m-2 \\ m\end{array}\right)<$ $2^{2 m-2}$. The sum over $D$ of the products over $l \in D$ is also easily accomplished, and 
one gets

$$
\sum_{D \in \mathscr{O}_{m-2}^{s}} \prod_{l \in D} \frac{1}{(1+|l|)^{1+d}}<(m-2) ! \kappa^{m-2}
$$

this corresponds to the fact that (with some overcounting) all diagrams of $\mathscr{D}_{m-2}$ can be obtained by diagrams of $\mathscr{D}_{m-3}$, by attaching to any of the $m-2$ vertices a line, whose free end runs freely over $\mathbf{Z}^{d}$. One then has

$$
\operatorname{mes}(\Omega)<\frac{\gamma}{\sigma \sqrt{\pi}} \sum_{s} \frac{1}{(1+|s|)^{1+d}} \sum_{m \geqq 3}\left(\frac{1}{2}\right)^{m-1}=\frac{1 \gamma \kappa}{2 \sigma \sqrt{\pi}},
$$

as claimed.

Let us now show how the Diophantine condition is used in our estimates. From (3.9) and (2.9) one has, by taking into account (3.13),

$$
\left\|\Phi_{m, D}^{s}\right\| \leqq \frac{\left\|\mathscr{R}_{m, D}^{s}\right\|}{\alpha_{m, D}^{s}}, \quad m \geqq 2
$$

In turn, this inequality, together with (3.12), gives rise to an iterative scheme, whose result, for any $s \in \mathbf{Z}^{d}, m \geqq 2, D \in \mathscr{D}_{m-1}^{s}$, is easily recognized to be

$$
\left\|\mathscr{R}_{m+1, D}^{s}\right\| \leqq 3^{m-1} m !\left\|\Phi_{2}^{s}\right\| \prod_{l \in D}\left\|V_{l}\right\| \Lambda_{m, D}^{s}
$$

where $\Lambda_{m . D}^{s}=1$ for $m=2$, and

$$
\Lambda_{m, D}^{s}=\sum_{l_{1}, \ldots, l_{m-1}} \frac{1}{\alpha_{m, D \backslash\left\{l_{1}\right\}}^{s} \cdots \alpha_{3, D \backslash\left\{l_{1}\right\} \cdot \backslash\left\{l_{m-2}\right\}}^{s}}
$$

for $m>2$, the sum being extended over the set

$$
\left\{l_{1}, \ldots, l_{m-1} \in D \subset \mathscr{D}_{m-1}^{s} ; D \backslash\left\{l_{1}\right\} \in \mathscr{D}_{m-2}^{s}, \ldots, D \backslash\left\{l_{1}\right\} \backslash \ldots \backslash\left\{l_{m-2}\right\} \in \mathscr{D}_{1}^{s}\right\} .
$$

Let us now use the expression (3.14) for $\alpha_{m, D}^{s}$. One has

$$
\begin{aligned}
\Lambda_{m, D}^{s}= & \gamma^{-m+2}(1+|s|)^{(d+1)(m-2)}(8 \kappa)^{(1 / 2)(m-1)(m-2)} \\
& \cdot(m-2) ! \cdots 1 ! \sum_{l_{1}, \ldots, l_{m-1}} \Xi_{l_{1}, \quad, l_{m-2}},
\end{aligned}
$$

with

$$
\begin{aligned}
\Xi_{l_{1}, \ldots, l_{m-2}} & =\left[\prod_{l \in D}(1+|l|)^{d+1}\right] \ldots\left[\prod_{l \in D \backslash\left\{l_{1}\right\} \cdot\left\{l_{l}-2\right\}}(1+|l|)^{d+1}\right] \\
& <\prod_{l \in D}(1+|l|)^{(d+1)(m-2)}
\end{aligned}
$$

One can then estimate the sum over $l_{1}, \ldots, l_{m-1}$ by a factor $(m-1)$ !, and use this result in (3.22), to get

$$
\begin{aligned}
\left\|\mathscr{R}_{m+1 . D}^{s}\right\|< & 3^{m-1} \gamma^{-m+2}(8 \kappa)^{(1 / 2) m(m-1)} m ! \ldots 2 ! \\
& \cdot(1+s)^{(d+1)(m-2)} \prod_{l \in D}\left\|V_{l}\right\|(1+|l|)^{(d+1)(m-2)}
\end{aligned}
$$


the factor $\left\|\Phi_{2}^{s}\right\|$ has been omitted, since, by its very definition, one has $\left\|\Phi_{2}^{s}\right\|=1$.

From this expression one sees that, in order to dominate the last factor, $\left\|V_{l}\right\|$ must decrease with $|l|$ more rapidly than any inverse power of $|l|$. It is then natural to assume

$$
\left\|V_{l}\right\| \leqq E e^{-K(1+|l|)^{\delta}}
$$

where $E$ and $K$ are arbitrary positive constants, and $0<\delta \leqq 1$. This is exactly what we assumed in Sect. $1, E$ depending on the constant $U$ introduced there. Using the well known inequality

$$
x^{\alpha} e^{-\beta x} \leqq \alpha^{\alpha} e^{-\alpha} \beta^{-\alpha},
$$

with $x=(1+|l|)^{\delta}, \alpha=(d+1)(m-2) / \delta, \beta=K / 2$, and the trivial estimate $2 ! \ldots m !<$ $m^{(1 / 2) m(m-1)}$, one gets

$$
\begin{aligned}
\left\|\mathscr{R}_{m+1 . D}^{s}\right\| \leqq & 3^{m-1} \gamma^{-m+2}(8 \kappa)^{(1 / 2) m(m-1)} m^{(1 / 2) m(m-1)}(1+|s|)^{(d+1)(m-2)} \\
& \cdot \prod_{l}\left[\left(\frac{d+1}{\delta}\right)^{((d+1) / \delta)(m-2)}\left(\frac{K e}{2}\right)^{-((d+1) / \delta)(m-2)} E e^{-(K / 2)(1+|l|)^{\delta}}\right] .
\end{aligned}
$$

Such expression can be given the form

$$
\left\|\mathscr{R}_{m+1, D}^{s}\right\| \leqq A_{0} B_{0}^{m} C_{0}^{m(m-1)} m^{(1 / 2) m(m-1)}(1+|s|)^{(d+1)(m-2)} \prod_{l} e^{-(K / 2)(1+|l|)^{\delta}},
$$

with suitable constants $A_{0}, B_{0}, C_{0}$, depending on $d, \delta, \gamma, K, E$.

\section{The Main Estimate}

As already remarked in the introduction, we are interested in studying the evolution of initial excitations localized around the (arbitrarily chosen) origin of our $d$-dimensional lattice. We thus consider initial data in the set $\mathscr{B}_{0}$ given by

$$
\left(p_{s}^{2}+q_{s}^{2}\right)^{1 / 2} \leqq \varepsilon \psi(|s|), \quad s \in \mathbf{Z}^{d},
$$

where $\psi(x)=(1+x)^{-d-1}$, and try to work out a lower bound for the time-scale at which the initial excitation starts to significantly evolve; say the time at which the orbit escapes the slightly larger domain $\mathscr{B}$, defined by

$$
\left(p_{s}^{2}+q_{s}^{2}\right)^{1 / 2} \leqq \sqrt{2} \varepsilon \psi(|s|), \quad s \in \mathbf{Z}^{d},
$$

i.e. by $\Phi_{2}^{s} \leqq \varepsilon^{2} \psi(|s|)^{2}$. Let us insert the latter inequality in our estimates. From the definition (2.5) of $x_{s}$ and $y_{s}$, it follows that, for $(p, q) \in \mathscr{B},\left|x_{s}\right|,\left|y_{s}\right|<\varepsilon \psi(|s|)$.

For a polynomial $f \in \Pi_{m}$, let us now introduce, besides the already considered norm $\|f\|$, the supremum norm in $\mathscr{B}$, precisely $\|f\|^{*}=\sup _{(p, q) \in \mathscr{B}}|f(p, s)|$. If $f \in \Pi_{m}$ has support $S \subset \mathbf{Z}^{d}$, then one clearly has that $\|f\|^{*} \leqq \varepsilon^{m} \psi(\eta)^{m}\|f\|$, with $\eta=\min _{s \in S}|s|$. From (3.31) one then concludes that

$$
\begin{aligned}
\left\|\mathscr{R}_{m+1, D}^{s}\right\|^{*} \leqq & \varepsilon^{m+1} \psi(\eta)^{m+1} A_{0} B_{0}^{m} C_{0}^{m(m-1)} \\
& \cdot m^{(1 / 2) m(m-1)}(1+|s|)^{(d+1)(m-2)} \prod_{l \in D} e^{-(K / 2)(1+|l|)^{\delta}},
\end{aligned}
$$

where $\eta=\min _{s^{\prime} \in \mathscr{S}(D)}\left|s^{\prime}\right|$. 
We now distinguish between two cases:

i. $\eta \geqq \frac{1}{2}|s|$. In this case one has $\psi(\eta) \leqq 2^{d+1} /(1+|s|)^{d+1}$, and consequently one obtains $(1+|s|)^{(d+1)(m-2)} \psi(\eta)^{m+1} \leqq 2^{(d+1)(m+1)} \psi(|s|)^{3}$.

ii. $\eta<\frac{1}{2}|s|$. In this case one cannot make any use of the factors $\psi(\eta)$; however, now one has that $\sum_{l \in D}|l| \geqq|s| / 2$, and consequently $\Pi_{l} e^{-(K / 2)(1+\mid l l)^{\delta}} \leqq e^{-(K / 4) \Sigma(1+\mid l l)^{\delta}}$ $\Pi_{l} e^{-(K / 4)(1+|l|)^{\delta}} \leqq e^{-(K / 4) 2^{-\delta}(1+|s|)^{\delta}} \quad \Pi_{l} e^{-(K / 4)(1+|l|)^{\delta}}$. Using the inequality (3.29), with $x=(1+|s|)^{\delta}, \alpha=\delta^{-1}(d+1)(m+1), \beta=2^{-\delta-2} K$, one obtains

$$
\begin{aligned}
& (1+|s|)^{(d+1)(m-2)} \prod_{l} e^{-(K / 2)(1+|l|)^{\delta}} \leqq(1+|s|)^{-3(d+1)} \\
& \quad \cdot\left[\frac{(d+1)(m+1)}{\delta}\right]^{(d+1)(m+1) / \delta}\left[2^{-\delta-2} K e\right]^{(d+1)(m+1) / \delta} \prod_{l \in D} e^{-(K / 4)(1+|l|)^{\delta}} .
\end{aligned}
$$

For $\mathscr{R}_{m+1, D}^{s}$, one obtains in both cases an estimate of the form

$$
\left\|\mathscr{R}_{m+1, D}^{s}\right\|^{*} \leqq \varepsilon^{m+1} \psi(|s|)^{3} A_{1} B_{1}^{m} C_{1}^{m(m-1)} m^{(1 / 2) m(m-1)} \prod_{l} e^{-(K / 4)(1+|l|)^{\delta}},
$$

with suitable constants $A_{1}, B_{1}, C_{1}$; (in particular, the change from $C_{0}$ to $C_{1}$ accounts for the leading term $(m+1)^{(d+1)(m+1) / \delta}$ in (4.4)).

Next we want to sum over $D \in \mathscr{D}_{m-1}^{s}$, to get our final estimate on $\left\|\mathscr{R}_{m+1}^{s}\right\|^{*}$. It is not difficult to recognize that one has

$$
\sum_{D \in \mathscr{O}_{m-1}^{s}} \prod_{l \in D} e^{-(K / 4)(1+|| \mid)^{\delta}}<m !\left[\sum_{s^{\prime} \in \mathbf{Z}^{d}} e^{-(K / 4)\left(1+\left|s^{\prime}\right|\right)^{\delta}}\right]^{m-1} ;
$$

this follows by induction, noting that all diagrams $D \in \mathscr{D}_{n}^{s}$ can be obtained from diagrams $D^{\prime} \in \mathscr{D}_{n-1}^{s}$, by attaching a line at any one of the $n$ vertices of $D^{\prime}$, and letting the other end, $s^{\prime}$, of $l$ range over $\mathbf{Z}^{d}$. Our basic estimate can then be given the form

$$
\left\|\mathscr{R}_{m+1}^{s}\right\|^{*} \leqq \varepsilon^{m+1} \psi(|s|)^{3} A_{2} B_{2}^{m} C_{2}^{m(m-1)} m^{(1 / 2) m(m-1)} .
$$

Moreover, one can find constants $F_{1}$ and $F_{2}$, such that, for any $m \geqq 3$, one has

$$
\begin{aligned}
m^{(1 / 2) m(m-1)} & \leqq F_{1}(m-3)^{(3 / 4)(m-3)^{2}}, \\
A_{2} B_{2}^{m} C_{2}^{m(m-1)} & \leqq F_{2}(m-3)^{(1 / 4)(m-3)^{2}}
\end{aligned}
$$

(for $m=3$, one sets $\left.0^{0}=1\right)$; the quantity $(m-3)$ has been introduced here for later convenience. Using (4.8), our estimate (4.7) for $\mathscr{R}_{m+1}^{s}$ takes the simpler form

$$
\begin{aligned}
\left\|\mathscr{R}_{m+1}^{s}\right\|^{*} & \leqq F \varepsilon^{3} \psi(|s|)^{3} \varepsilon^{m-2}(m-3)^{(m-3)^{2}} \\
& =F \varepsilon^{3} \psi(|s|)^{3} e^{-\left[(m-2) \ln \varepsilon^{-1}-(m-3)^{2} \ln (m-3)\right]},
\end{aligned}
$$

where $F=F_{1} F_{2}$. This constant $F$ depends on all constants entering the problem, more precisely on $d, \delta, \gamma, K, E$.

Let us now choose $m=M$, in order to arrive at an estimate for our final remainder $\mathscr{R}_{M+1}^{s}$. At this point we introduce the basic analytic idea underlying Nekhoroshev estimates: We let $M$ be a suitable function of $\varepsilon$, such that $M \rightarrow \infty$, as $\varepsilon \rightarrow 0$. The idea is to choose the $\varepsilon$-dependence of $M$ in such a way, that, for each $\varepsilon$, the right-hand side of (4.9) is as small as possible. Here we find an important 
difference in comparison with the finite dimensional case: Instead of taking $M$ to be an inverse power of $\varepsilon$, the best we can do here is to let $M$ be of order $\ln \varepsilon^{-1} / 2 \ln \ln \varepsilon^{-1}$; a convenient choice is

$$
\frac{\ln \varepsilon^{-1}}{2 \ln \ln \varepsilon^{-1}}-1<M-3 \leqq \frac{\ln \varepsilon^{-1}}{2 \ln \ln \varepsilon^{-1}},
$$

which yields, as is checked immediately,

$$
(M-2) \ln \varepsilon^{-1}-(M-3)^{2} \ln (M-3) \geqq \frac{1}{4} \frac{\left(\ln \varepsilon^{-1}\right)^{2}}{\ln \ln \varepsilon^{-1}} .
$$

One then has the estimate

$$
\left\|\mathscr{R}_{M+1}^{s}\right\| \|^{*} \leqq F \varepsilon^{3} \psi(|s|)^{3} e^{-(1 / 8)\left(\ln e^{-1}\right)^{2} \ln \ln e^{-1}},
$$

which is much worse than Nekhoroshev exponentials. Yet, for $\varepsilon \rightarrow 0$, it tends to 0 faster than any power of $\varepsilon$. Let us recall that this estimate is correct for $(p, q) \in \mathscr{B}$, i.e., provided one has

$$
\Phi_{2}^{s}<\varepsilon^{2} \psi(|s|), \quad s \in \mathbf{Z}^{d}
$$

Next we need an estimate for $\Phi^{\mathrm{s}, M}-\Phi_{2}^{\mathrm{s}}=\sum_{m=3}^{M} \Phi_{m}^{s}$. Following almost literally the estimates for $\mathscr{R}_{m}^{s}$, it is not difficult to get

$$
\left\|\Phi_{m}^{s}\right\| * \leqq \varepsilon^{m} \psi(s)^{3} G(m-3)^{(m-3)^{2}}, \quad m \geqq 3,
$$

$G$ being a suitable constant, and thus

$$
\begin{aligned}
\left\|\Phi^{s, M}-\Phi_{2}^{s}\right\|^{*} & \leqq G \varepsilon^{3} \psi(s)^{3} \sum_{m=3}^{M}\left(\varepsilon(m-3)^{m-3}\right)^{m-3} \\
& \leqq G \varepsilon^{3} \psi(s)^{s} \sum_{m=0}^{M-3}\left(\varepsilon(M-3)^{M-3}\right)^{m} .
\end{aligned}
$$

Since $M-3 \leqq\left(\ln \varepsilon^{-1}\right) /\left(2 \ln \ln \varepsilon^{-1}\right)$, one has $(M-3)^{M-3}<\varepsilon^{-1 / 2}$, so that, for $\varepsilon<\frac{1}{4}$, say, one finds

$$
\left\|\Phi^{s, M}-\Phi_{2}^{s}\right\|^{*} \leqq 2 G \varepsilon^{3} \psi(|s|)^{3} .
$$

Now we choose initial data $\left(p^{0}, q^{0}\right)$ satisfying (4.1), i.e., $\Phi_{2}^{s}\left(p^{0}, q^{0}\right) \leqq$ $\left(\varepsilon^{2} / 2\right) \psi(|s|)^{2}$. As far as the orbit $p(t), q(t)$ remains in $\mathscr{B}$, as given by (4.2), we can use our estimates (4.12) and (4.16). We note that

$$
\begin{aligned}
\Phi_{2}^{s}(p(t), q(t))-\Phi_{2}^{s}\left(p^{0}, q^{0}\right)= & \Phi_{2}^{s}(p(t), q(t))-\Phi^{s, M}(p(t), q(t)) \\
& +\Phi^{s, M}(p(t), q(t))-\Phi^{s . M}\left(p^{0}, q^{0}\right) \\
& +\Phi^{s, M}\left(p^{0}, q^{0}\right)-\Phi_{2}^{s}\left(p^{0}, q^{0}\right),
\end{aligned}
$$

and use (4.12), (4.16) to estimate the right-hand side. that

Recalling that $\dot{\Phi}^{s, M}=\left\{H, \Phi^{s, M}\right\}=\Re_{M+1}^{s}$, one concludes from (4.17) and (4.16)

$$
\left|\Phi_{2}^{s}(p(t), q(t))-\Phi_{2}^{s}\left(p^{0}, q^{0}\right)\right| \leqq 4 G \varepsilon^{3} \psi(|s|)^{3}+|t|\left\|\mathscr{R}_{M+1}^{s}\right\|^{*}
$$


Thus, for $t$ satisfying

one has

$$
|t| \leqq \frac{G}{F} e^{(1 / 8)\left(\ln \varepsilon^{-1}\right)^{2} / \ln \ln \varepsilon^{-1}}
$$

$$
\left|\Phi_{2}^{s}(p(t), q(t))-\Phi_{2}^{s}\left(p^{0}, q^{0}\right)\right| \leqq 5 G \psi(s)^{3} \varepsilon^{3},
$$

i.e., the initial excitation essentially does not evolve. Let us notice that, for $\varepsilon<(10 G)^{-1}$, one has from (4.20) $\left|\Phi_{2}^{s}(p(t), q(t))\right|<\varepsilon^{2} \psi(s)^{2}$, so that $(p(t), q(t)) \in \mathscr{B}$, as required for consistency.

The inequalities (4.19) and (4.20) are our final result and coincide with (1.12), (1.13) of the theorem stated in Sect. 1, provided one sets $\mathscr{G}=5 G$ and $\mathscr{T}=G / F$.

\section{Summary and Outlook}

In this paper, we have established a Nekhoroshev-type estimate for some Hamiltonian systems with infinitely many degrees of freedom which are anharmonic perturbations of infinite systems of uncoupled, non-resonant harmonic oscillators. The anharmonic perturbation couples finite subsets of oscillators; the couplings decrease rapidly in the cardinality and the size of those subsets. For models in which the frequencies of the harmonic oscillators are independent, identically distributed random variables with smooth, e.g. Gaussian distribution, we have shown that our non-resonance conditions on the frequencies hold with large probability. The main result is that if the non-resonance conditions hold we can control motions of our systems with localized initial conditions, in the sense of inequality (1.12), up to times, $T_{\varepsilon}$, of order $\exp \left[\right.$ const. $\left.(\ln \varepsilon)^{2} / \ln \ln \varepsilon^{-1}\right]$, where $\varepsilon$ measures the strength of the anharmonic perturbation (for the given initial condition).

Such motions are close to integrable motions and essentially remain localized in a bounded lattice region, in the sense of inequality (1.13), up to times $\leqq T_{\varepsilon}$.

Our result suggests that, in such systems, transport of energy is anomalously slow. But, in order to establish transport properties of our systems, we would have to control their motions over arbitrarily long time spans and relax our non-resonance conditions. We would have to admit configurations of oscillator frequencies in our analysis with the property that small, dilute subsystems of oscillators are at resonance. It is expected that the large region of non-resonant oscillators surrounding small subsystems at resonance will still provide an effective barrier preventing rapid transport of energy. Such a situation has been studied in [13], but, in that paper, motions are controlled only up to times of order $\varepsilon^{-1}$ (which makes transport properties entirely inaccessible).

Nearly resonant subsystems appear in the analysis of [3], and the results in [3] provide control over motions on arbitrarily long time scales. But the dynamics of the systems studied in [3] is linear. This has a very important consequence: the location of nearly resonant subsystems is time-independent. In contrast, if the dynamics is non-linear, resonant subsystems may be born and disappear again, as time evolves. We do not know any methods that would yield control over the disappearance of resonant situations in finite subsystems, as time evolves. 
A drawback of most currently known methods is that they attempt to control the motion of a system on a "microscopic level." For our goal of establishing transport properties of Hamiltonian systems with infinitely many degrees of freedom we should pass to "coarse-grained" descriptions of the dynamics of finite subsystems, based on following the motion of collective degrees of freedom. Our description ought to become more and more coarse-grained, as time grows. This would render resonant situations in small subsystems more and more "invisible." This is the idea behind the analysis in [3]. It is reminiscent of the renormalization group strategy, but we do not know how to implement it in practice for non-linear dynamical systems. We thus lose control over the motions of our systems after large, but finite times, (although existence of dynamics for all times is usually easy to prove).

Our methods could be applied to translation-invariant systems of plane rotors weakly coupled by forces of short range. Thanks to the anisochronicity condition satisfied by the unperturbed Hamilton function resonances can be avoided by a suitable choice of initial conditions of finite total energy. See also $[13,14]$ for results concerning systems of coupled rotors.

Control of transport properties at positive temperature (initial conditions with finite energy density, but infinite total energy) looks largely inaccessible at present.

Acknowledgements. We gratefully acknowledge helpful discussions with T. Spencer and C. E. Wayne on a variety of related problems. We thank the Fonds National Suisse for financial support.

\section{References}

1. Anderson, P. W.: Phys. Rev. 109, 1492 (1958)

2. Wegner, F.: Z. Phys. B 22, 273 (1975); Gol'dsheid, Ya., Molchanov, S., Pastur, L.: Funct. Anal. Appl. 11, 1 (1977); Kunz, H. Souillard, B.: Commun. Math. Phys. 78, 201 (1980); Delyon, F., Kunz, H. Souillard, B.: J. Phys. A 16, 25 (1983); Carmona, R.: Duke Math. J. 49, 191 (1982)

3. Fröhlich, J., Spencer, T.: Commun. Math. Phys. 88, 151 (1983); Fröhlich, J., Martınelli, F., Scoppola, E., Spencer, T.: Commun. Math. Phys. 101, 21 (1985); Simon, B., Wolff, T.: Commun. Pure Appl. Math. 39, 75 (1986); Delyon, F., Lévy, Y., Souillard, B.: Commun. Math. Phys. 100, 463 (1985)

4. Fröhlich, J., Spencer, T., Wayne, C. E.: J. Stat. Phys. 42, 247 (1986)

5. Nekhoroshev, N. N.: Usp. Mat. Nauk 32, (1977) [Russ. Math. Surv. 32, 1 (1977)]

6. Nekhoroshev, N. N.: Trudy Sem. Petrows. No. 5, 5 (1979)

7. Benettin, G., Galgani, L., Giorgilli, A.: Celestial Mech. 37, 1 (1985)

8. Gallavottı, G.: Quasi-integrable mechanical systems. In: Critical Phenomena, Random Systems, Gauge Theories, Osterwalder, K., Stora, R. (eds), Les Houches, Session XLIII, 1984, Amsterdam: Holland 1986

9. Giorgilli, A., Galgani, L.: Celestial Mech. 37, 95 (1985)

10. Benettin, G., Gallavotti, G.: J. Stat. Phys. 44, 293 (1985)

11. Diana, E., Galgani, L., Giorgilli, A., Scotti, A.: Boll. Union Mat. Ital. 11, 84 (1975)

12. Giorgilli, A.: Rigorous Results on the Power Expansions for the Integrals of a Hamiltonian System near an Elliptic Equilibrium Point, preprint

13. Wayne, C. E.: Commun. Math. Phys. 103, 351 (1986) and 104, 21 (1986)

14. Bellissard, J., Vittot, M.: Preprint, Marseille 1985

Communicated by A. Jaffe

Received May 13, 1988 